\title{
Correction to: Understanding the Bariatric Patient Perspective in the National Patient-Centered Clinical Research Network (PCORnet) Bariatric Study
}

\author{
Karen J. Coleman ${ }^{1} \cdot$ David G. Schlundt ${ }^{2} \cdot$ Kemberlee R. Bonnet $^{2} \cdot$ Kimberly J. Holmquist $^{1} \cdot$ Jennifer Dunne ${ }^{3}$. \\ Elizabeth Crull $^{4}$ • Beatrice Y. Hanaoka ${ }^{5}$. Michelle R. Lent ${ }^{6}$. Joe Nadglowski ${ }^{7}$. Louisa G. Sylvia ${ }^{8}$. \\ Shilpa Venkatachalam ${ }^{9}$. Stavra A. Xanthakos ${ }^{10} \cdot$ Roni Zeiger $^{11} \cdot$ David Arterburn $^{12} \cdot$ Neely Williams $^{13}$. \\ Anita Courcoulas ${ }^{14}$. Jane Anau ${ }^{12} \cdot$ Kathleen M. McTigue ${ }^{15}$ - for the PCORnet Bariatric Study Collaborative
}

Published online: 16 February 2020

(C) Springer Science+Business Media, LLC, part of Springer Nature 2020

\section{Correction to: Obesity Surgery. https://doi.org/10.1007/s11695-020-04404-8}

In the original article the list of author names and affiliations was incorrect. They are correct here.

*PCORnet Bariatric Study Collaborative:

Cynthia Blalock, Vanderbilt University Medical Center, Nashville, TN.

Elisha Malanga, COPD Foundation, Miami, FL.

Joseph Vitello, Jesse Brown VA Medical Center, Chicago, IL.

Tammy St. Clair, (no institution), New York, NY.

Julie Tice, (no institution), Hershey, PA.

James C. McClay, University of Nebraska Medical Center, Omaha, NE.

Corrigan L. McBride, University of Nebraska Medical Center, Department of Surgery, Omaha, NE.

Ana Emiliano, Columbia University, New York, NY.

The online version of the original article can be found at https://oi.org/ 10.1007/s11695-020-04404-8

Karen J. Coleman

Karen.J.Coleman@kp.org

1 Department of Research and Evaluation, Kaiser Permanente Southern California, Pasadena, CA, USA

2 Department of Psychology, Vanderbilt University, Nashville, TN, USA

3 Smart Patients, San Jose, CA, USA

4 Louisiana Public Health Institute, New Orleans, LA, USA

5 School of Medicine, University of Alabama, Birmingham, AL, USA

6 Philadelphia College of Osteopathic Medicine, Philadelphia, PA, USA
Rabih Nemr, Lutheran Medical Center, New York, NY.

Marc P. Michalsky, Nationwide Children's Hospital, Columbus, $\mathrm{OH}$.

Sameer Murali, Kaiser Permanente Southern California, Center for Healthy Living, Fontana, CA.

Ali Tavakkoli, Brigham and Women's Hospital, Boston, MA. Nirav K. Desai, Boston Children's Hospital, Boston, MA.

Caroline M. Apovian, Boston University School of Medicine and Boston Medical Center, Boston, MA.

Jeanne M. Clark, Johns Hopkins University and Health Plan, Baltimore, MD.

Elizabeth Nauman, Louisiana Public Health Institute, New Orleans, LA.

Elizabeth Cirielli, Brigham and Women's Hospital Boston, Boston, MA.

Cheri D. Janning, Duke Clinical Science Translational Institute, Durham, NC.

7 Obesity Action Coalition, Tampa, FL, USA

8 Massachusetts General Hospital, Boston, MA and Harvard Medical School, Cambridge, MA, USA

9 Global Healthy Living Foundation, New York, NY, USA

10 Cincinnati Children's Hospital Medical Center, Cincinnati, OH, USA

11 Facebook Inc, Mountainview, CA, USA

12 Health Research Institute, Kaiser Permanente Washington, Seattle, WA, USA

13 Community Partners Network, Nashville, TN, USA

14 University of Pittsburgh Medical Center, Pittsburgh, PA, USA

15 University of Pittsburgh, Pittsburgh, PA, USA 